\title{
LETTER TO THE EDITOR KSHV-encoded vIL-6 collaborates with deregulated c-Myc to drive plasmablastic neoplasms in mice
}

Blood Cancer Journal (2016) 6, e398; doi:10.1038/bcj.2016.6; published online 26 February 2016

The mechanism by which Kaposi sarcoma (KS)-associated herpesvirus (KSHV), now recognized as human HV-8 (HHV-8), drives cancer is poorly understood. ${ }^{1}$ In addition to KS, the virus causes lifethreatening pathologies of the mature B-lymphocyte lineage, ${ }^{2}$ including multi-centric Castleman's disease (MCD), ${ }^{3}$ diffuse large B-cell lymphoma evolving from MCD (MCD-DLBCL) ${ }^{4}$ and primary effusion lymphoma (PEL). ${ }^{5}$ These B-cell disorders are a significant public health concern because KSHV is frequently associated with HIV, which by itself increases the risk of developing B-lymphoma by $\sim 2$ orders of magnitude. In addition, the broad implementation of highly effective antiretroviral therapy has inadvertently caused a spike in lymphoma-related morbidity and mortality due to increased survival of HIV patients. Here, we took advantage of the laboratory mouse to enhance our understanding of KSHV-dependent pathophysiology in the B-lymphocyte lineage. Specifically, we evaluated the hypothesis that a KSHV-encoded cytokine, viral interleukin-6, hereafter called vIL- 6 , may be a driver of the transformation of B-lymphocytes to a malignant state. Our experimental strategy included the refinement of a recently developed C57BL/6 (B6) mouse model of constitutive, $\mathrm{H} 2-\mathrm{K}$ promoter-driven, transgenic (TG) vlL- 6 expression ${ }^{6}$ by making two critical changes. First, we backcrossed the vlL6 transgene from the genetic background of $B 6$ onto $B A L B / C(C)$, an inbred strain of mice that is hyper-susceptible to malignant plasma cell tumors, such as inflammation-dependent peritoneal plasmacytoma. ${ }^{7}$ Second, we intercrossed the newly generated C.vlL6 congenic mice with C.iMyc $\Delta \mathrm{E} \mu$ mice, a gene-insertion model of the chromosomal translocation $T(12 ; 15)$ that results in the deregulated expression of the cellular oncogene $M y c$ in the B-cell lineage. We found that single-TG C.vlL6 mice are prone to a severe and sometimes fatal MCD-like disease, whereas double-TG C.vIL6iMyc mice invariably developed aggressive plasmablastic neoplasms that exhibited striking clinical and histopathological similarities to human PEL, plasmablastic lymphoma (PBL) and immunoglobulin (Ig)-producing, extramedullary plasmablastic plasma cell myeloma (PBM). The new findings will be briefly summarized below.

We previously reported that vIL6-TG B6 mice (i) exhibited vlL-6 serum levels comparable to those observed in KSHV-infected patients, (ii) contained elevated amounts of pSTAT3 in lymphoid tissues and (iii) developed, in dependency on mouse IL-6 (mIL-6), MCD-like changes with low genetic penetrance and late onset. However, malignant tumors were not seen. ${ }^{6}$ To assess the suitability of the vlL6 transgene for modeling KSHV-associated lymphoma, we first asked whether the transgene distorts the composition of the cell compartment in which malignant transformation is postulated to occur: mature B-lymphocytes. This is an important consideration for tumor studies using TG mice, because cancer may be the indirect outcome of developmental aberrations of the cell lineage that undergoes neoplastic transformation, the direct consequence of the oncogenic properties of the TG driver or a combination of both. Our analysis of the mature splenic B-cell compartment in vIL6-TG B6 mice demonstrated that the frequency of follicular, marginal zone and transitional B cells was comparable to that in age-matched normal B6 mice (Figure 1a). Non-immunized vIL6-TG mice did not exhibit spontaneous germinal centers (Figure $1 \mathrm{~b}$ ) or changes in the $\mathrm{lg}$ isotype profile of $\mathrm{B}_{2} 20^{+} \mathrm{PNA}^{+} \mathrm{B}$ cells (Figure 1c) compared with controls. Likewise, flow cytometric analysis of vlL6-TG mice demonstrated no increase in $\mathrm{CD} 138^{+} \mathrm{B} 220^{+}$plasmablasts or $\mathrm{CD} 138^{+} \mathrm{B} 220^{-}$ plasma cells in the spleen (Figure 1d) or other lymphoid tissues (not shown). These results agreed with a wholesale lack of lymph node enlargement and splenomegaly on necropsy of 10 randomly chosen mice $\sim 8$ months of age (not shown) and supported the view that the vlL6 transgene on the genetic background of B6 is a weak oncogene that renders this strain of mice impractical for cancer studies.

We transferred the vlL6 transgene to the genetic background of $C$ (by means of introgressive backcrossing for 10 consecutive generations) to increase the efficacy with which enforced expression of vlL-6 drives malignant B-cell transformation (Figure 1e). The rationale for this approach was twofold. First, owing to a complex genetic trait that is determined by susceptibility genes in both the B-lymphocyte lineage (for example, Cdkn2a and Mtor) and the tumor microenvironment (for example, Mndal), C mice are highly susceptible to neoplasms of plasmablasts and plasma cells ${ }^{8}$ - key constituents of KSHV-related B-cell disorders. Second, a previous study from our laboratory showed that the B6-to- $\mathrm{C}$ transfer of a human IL-6 transgene-controlled by a MHC-I promoter $\left(\mathrm{H}-2 \mathrm{~L}^{\mathrm{d}}\right)$ that is very similar to the one driving vlL-6 expression $(\mathrm{H}-2 \mathrm{~K})$-resulted in a dramatic phenotypic shift from benign hyperplasia (plasmacytosis) to malignant growth (plasmacytoma), ${ }^{9}$ thus providing a compelling precedent for the impact of the $C$ genotype on vIL-6-dependent tumor formation. We generated a small cohort of C.vlL- $6 \mathrm{~N}_{10}$ mice ( $n=10)$, observed them to 250 days of age and found that these mice developed MCD-like plasma cell proliferations faster (mean onset $219 \pm 14.6$ days) and with higher incidence $(7 / 10,70 \%)$ than their B6 counterparts $(7 / 25,28 \%)^{6}{ }^{6}$ Disease progression was evident by gross pathological changes such as splenomegaly (mean weight $337 \pm 220 \mathrm{mg}$ ) and general lymphadenopathy (Figure 1f) and by histopathologic changes including expansive accumulations of plasma cells in lymph nodes (Figure 1g), occurrence of abundant $\mathrm{IgM}^{+}$plasma cells in inter-follicular splenic areas (Supplementary Figure 1a), increased numbers of megakaryocytes in medullary and extramedullary tissues (Supplementary Figure 1b) and aggregates of plasma cells at sites where cells of this sort normally do not occur (Supplementary Figure 1c). A single C.vlL-6 mouse developed a neoplasm of plasmablasts that resembled human extracavitary PEL/PBL (not shown). Serum protein electrophoresis of 6 C.vlL- 6 mice 215 days of age uncovered polyclonal hyper-gammaglobulinemia and monoclonal extragradients (M-spikes) in three and two cases, respectively (Figure $1 \mathrm{~h}$, Supplementary Figure $2 \mathrm{a}$, left). These findings demonstrated the amplification effect of $C$ alleles on the oncogenic potency of vlL- 6 in plasmablasts and plasma cells and indicated that the C.vlL6 model of human MCD (almost universally fatal if untreated ${ }^{10}$ ) is superior to the parental B6 model.

Recent approaches to improve the treatment of KSHV-associated B-cell disorders have focused on MYC ( $v$-myc myelocytomatosis viral oncogene homolog) as a molecular target. ${ }^{11}$ MYC is also involved in the natural history of MCD-DLBCL and PEL; for example, by regulating KSHV's latent and lytic life cycles. ${ }^{12}$ On this backdrop, we 
a

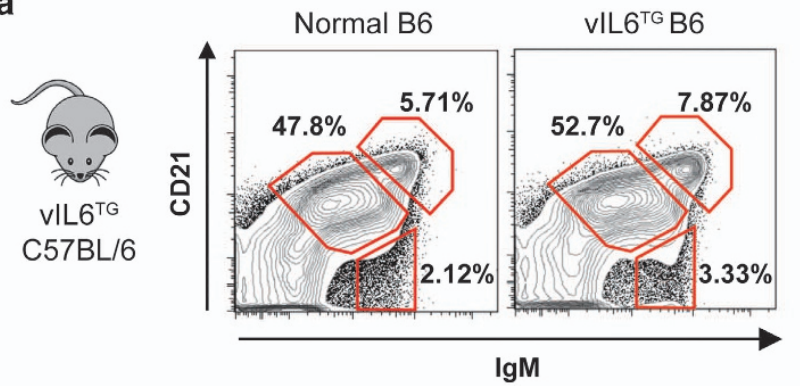

b

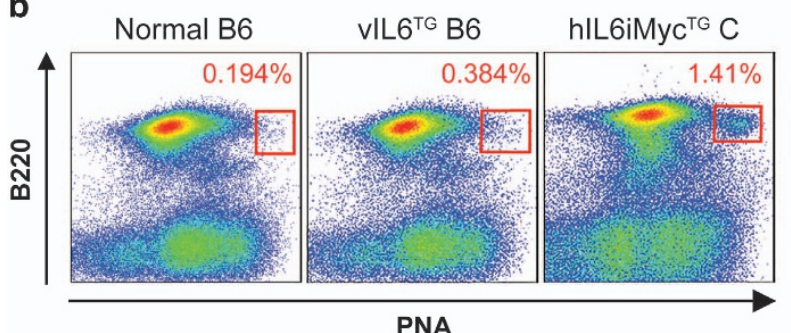

C

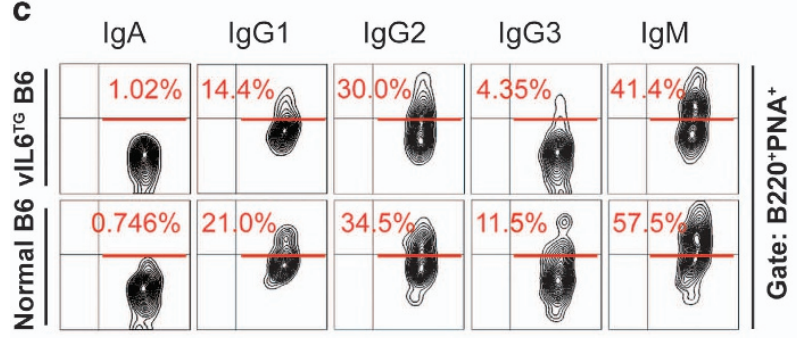

d Normal B6 (2 mo) vIL6 ${ }^{\mathrm{TG}} \mathrm{B} 6(2 \mathrm{mo}) \mathrm{vlL}^{\mathrm{TG}} \mathrm{B} 6(8 \mathrm{mo})$

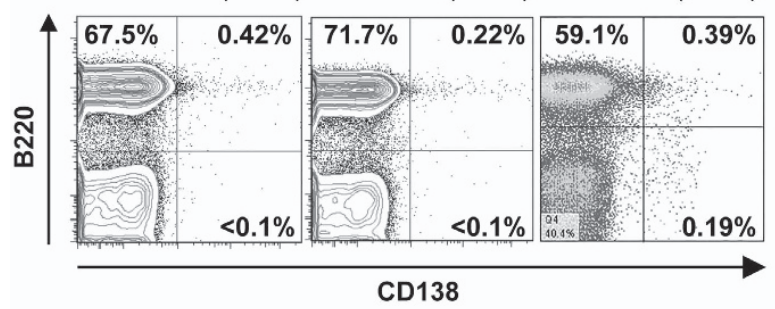

e

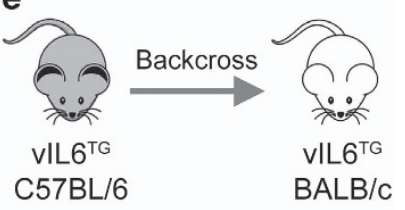

f

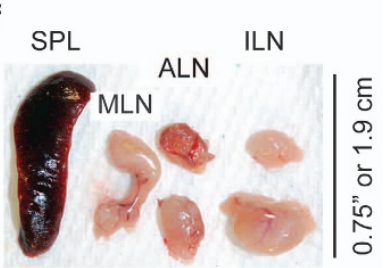

g

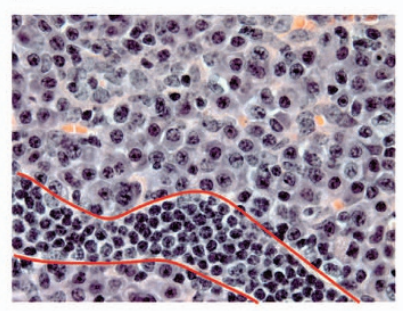

h

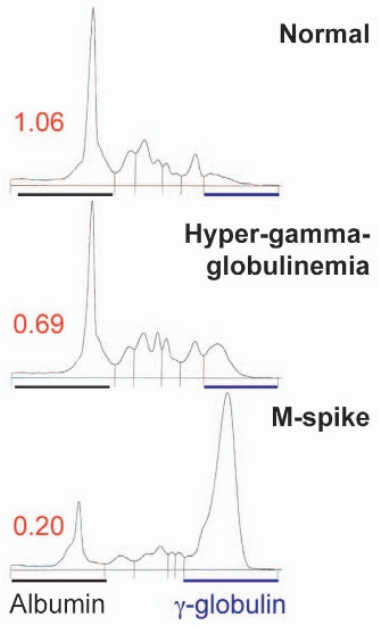

Figure 1. B6-to-C transfer of transgenic vlL-6 expression exacerbates MCD-like disease in mice. (a) Splenic B-cell compartments in vIL6-TG B6 mice are normal. Splenocytes were gated on B220 (CD45) and labeled with antibodies to CD21 and lgM to enumerate follicular (CD21 ${ }^{+}$IgM ${ }^{l o}$ ), transitional $\left(\mathrm{CD} 21^{-} \mathrm{IgM}^{\mathrm{hi}}\right)$ and marginal zone $\left(\mathrm{CD} 21^{\mathrm{hi}} \mathrm{IgM}^{\mathrm{hi}}\right) \mathrm{B}$ cells. The frequency of these cells was comparable in age-matched vIL6-TG and normal mice. (b) Normal numbers of spontaneous germinal center (GC) B cells in vlL6-TG B6 mice. Splenocytes were labeled with antibodies to B220 and PNA (peanut agglutinin) to enumerate GC B cells (red rectangles). The frequency of these cells was comparably low in vlL6-TG and normal B6 mice, but elevated in C mice that harbored a human IL6 and mouse Myc transgene (hIL6iMyc). ${ }^{14}$ The latter were included as positive control. (c) Normal isotype profile of GC B cells in vlL6-TG B6 mice. Splenocytes, gated on B220 and PNA, were labeled with antibodies to the indicated isotypes (immunoglobulin heavy-chains) expressed on the cell surface. Percentages of cells that express a given isotype are indicated in red. Shown is a representative result from independent measurements, using different, age-matched transgenic and normal mice. The immunoglobulin expression pattern was comparable in the two strains of mice. (d) Low numbers of splenic plasmablasts (PBs) and plasma cells (PCs) in vlL6-TG B6 mice. Forward scatter- and side scatter-gated splenocytes were evaluated for the expression of B220 and CD138 (syndecan-1) to distinguish $B 220^{+}$CD $138^{-}$B cells and B220- CD138 ${ }^{+}$PCs. Normal mice (used as control, left panel) and age-matched vIL6-TG mice (center) contained $<0.1 \%$ PCs. Eight-month-old transgenic mice (right) exhibited a small but reproducible increase in PCs that can be attributed to aging. (e) Generation of C.vlL6 congenic mice. The vlL6 transgene was transferred from B6 to C by 10 generations of introgressive backcrossing. (f) Splenolymphadenopathy due to plasmablastic MCD-like disease in C.vIL-6 mice. Shown is a photomicrograph of the enlarged spleen ( 350 mg, left), mesenteric lymph node (MLN) and left and right axillary (ALN) and inguinal (ILN) lymph nodes from a diseased mouse. (g) Histopathologic examination of one of the ILNs shown in $\mathbf{f}$ reveals an abnormal accumulation of aberrant PCs in medullary cords. A streak of small lymphocytes located at the bottom (indicated by two red lines) is included for size comparison (H\&E, $63 \times$ ). (h) Elevation of serum lg levels in 215-day-old C.vIL-6 mice. Shown are densitographic representations of the abundance of serum proteins fractionated by electrophoresis. Albumins and $\gamma$-globulins are indicated by black and blue horizontal lines, respectively. A mouse exhibiting hyper-gammaglobulinemia (center) or M-spike (bottom) is compared with an age-matched normal C mouse (top). The ratios of albumin to $\gamma$-globulin are indicated by red numbers. H\&E, hematoxylin and eosin. 

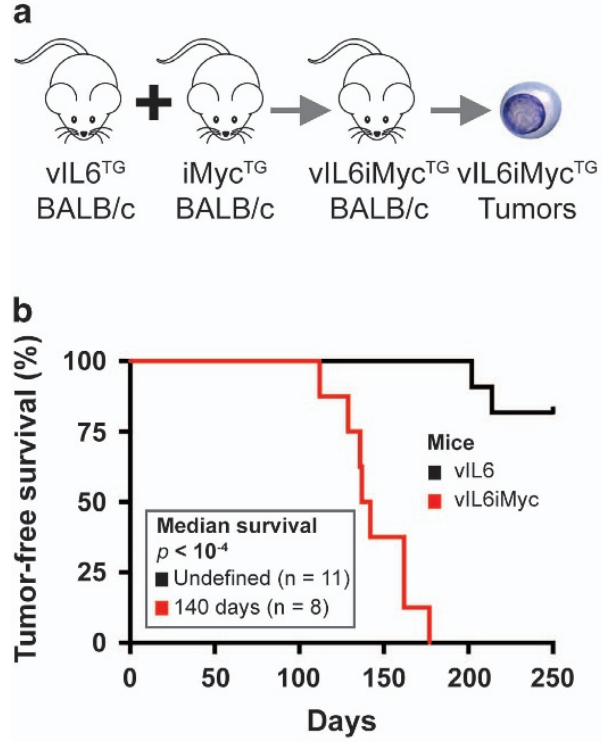

C

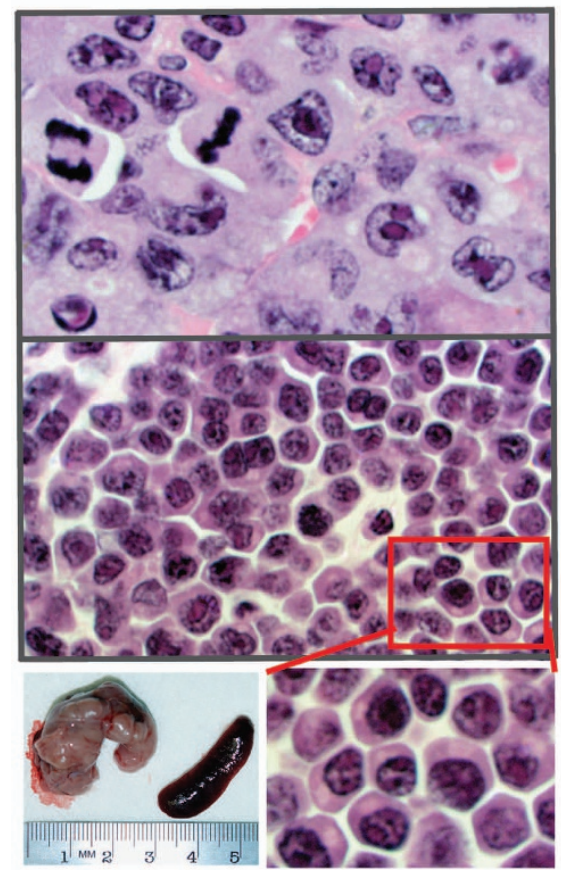

Figure 2. See caption for next page

decided to investigate whether vlL-6 collaborates with deregulated Myc in lymphoma development in mice. We generated a pilot cohort of double-TG C.vlL6iMyc mice $(n=7)$ (Figure 2a) and determined their tumor-free survival. Tumor development was fully penetrant (100\% tumor incidence) and tumor onset was short (140 days median) and predictable (110-160 days range; Figure $2 b$ ). Histopathological examination showed predominantly plasmablastic neoplasms that recapitulated many features of human PBL and/ or extramedullary PBM (Figure 2c, top), although some areas contained plasma cell neoplasms similar to the extensive sheets of aberrant plasma cells seen in MCD-bearing C.vIL-6 mice (Figure 2c, center). The malignant plasma cells were readily detected by flow cytometry using antibody to CD138 (Figure 2d). Serum samples of four out of four tumor-bearing mice contained massive M-spikes that caused up to fivefold drops in albumin-to-globulin ratios (Figure 2e; Supplementary Figure 2a, right). Of note, three out of d

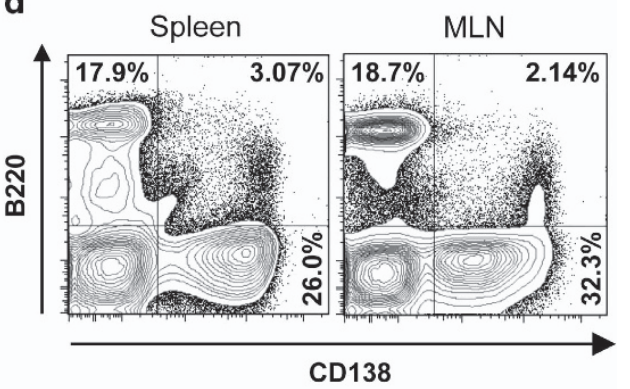

e

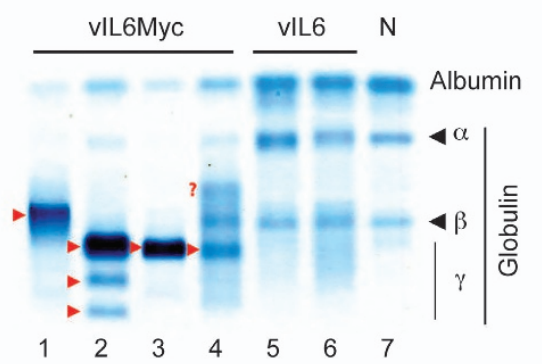

$\mathbf{f}$

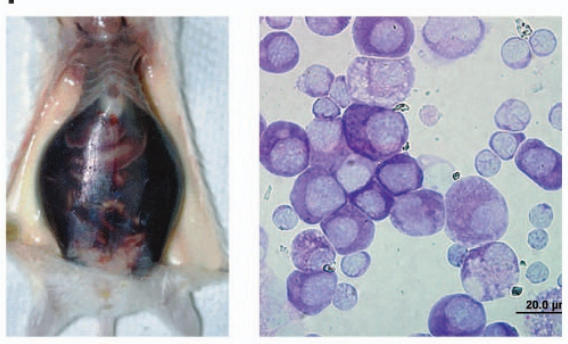

g

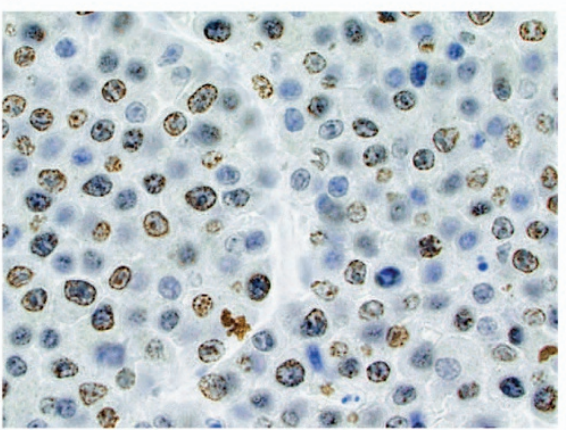

seven mice demonstrated tumor cell-laden cavitary effusions that resembled human PEL (Figure 2f). These tumors were as highly proliferative as the PBL-like neoplasm depicted in Figure 2g, using Ki67 immunoreactivity as measurement tool (up to $80 \% \mathrm{Ki}^{6} 7^{+}$cells, not shown). We concluded that strain C.vlL6iMyc is prone to a spectrum of neoplasms that mimic features of human KSHV-associated malignancies including PEL. Furthermore, the iMyc transgene, which accelerated vlL6-dependent disease, may serve as surrogate of human MYC for preclinical studies on MYC-targeted therapies of KSHVassociated lymphoma.

The principal advance described in this Letter is improved preclinical modeling of vlL6-dependent pathophysiologies in the mature B-cell lineage. Strain C.vlL6 and C.vlL6iMyc mice may be useful for translational studies on new approaches to treat and prevent KSHV-associated, vIL-6-driven B-cell disorders more effectively. For example, clinical observations indicating that 
Figure 2. Double-TG C.vIL6iMyc mice are prone to malignant B-cell tumors associated with KSHV in humans. (a) Schematic overview of generating C.vlL6iMyc-TG mice and observing them for tumor development. Heterozygous TG vlL6 mice were intercrossed with homozygous-TG iMyc mice. Double-TG vIL6iMyc mice, which occurred at the expected frequency of $\sim 50 \%$, were identified by PCR-based genotyping. The mice were left untreated and observed for tumor manifestation. Neoplastic tissues were analyzed using flow cytometric and histopathological methods. (b) Line graphs indicating tumor-free survival of single-TG vlL6 mice (black, $n=11)$ and double-TG vIL6iMyc mice (red, $n=8$ ). Median tumor-free survival of vlL6 mice (undefined at 250 days of age) was significantly longer than that of vlL6iMyc mice (140 days), using Mantel-Cox log-rank analysis $\left(P<10^{-4}\right)$. Single TG C.iMyc mice are not included in the graph because spontaneous tumors are rare and tumor-free survival has not been determined yet. In a previous study, we found that 8 of 76 (10.5\%) mice had developed tumors by 410 days of age (median: 251 days; mean: $269 \pm 64.3$ days; range: $213-410$ days). ${ }^{14}$ Thus, although definitive data are not available at this juncture, C.iMyc mice appear to be even less prone to malignancy than C.vIL6Myc mice are. (c) Representative $\mathrm{H} \& \mathrm{E}$-stained tissue section of a plasmablastic neoplasm (top, original magnification $\times 100$ ) and plasma cell proliferation (center, original magnification $\times 100)$. Both lesions were obtained from the same C.vlL6iMyc mouse. The plasmablastic neoplasm showed multi-organ involvement including bone marrow, enlarged lymph nodes, liver, spleen and full thickness small bowel tumors. The plasma cell proliferation was observed in focal areas of mesenteric fat and peri-nephric tissue. The greatly enlarged mesenteric lymph node, which was effaced by the plasmablastic neoplasm, is shown at the bottom left. (d) Flow cytometric histograms of a representative plasmablastic neoplasm. The tumor arose in a vlL6iMyc mouse at 130 days of age. Forward scatter- and side scatter-gated cells from spleen (left) or mesenteric lymph node (MLN, right) were evaluated for the expression of B220 (B-cell marker) and CD138 (syndecan-1, plasma cell marker). Nearly one-third of cells in both tissues were B220-CD138+ plasma cells. (e) Serum protein electropherograms of four different tumor-bearing C.vIL6iMyc mice (lanes 1-4), two C.vIL6 mice with incipient MCD-like disease (lanes 5-6) and one age-matched normal (N) control (lane 7). M-spikes (paraproteins) are indicated by red arrowheads pointing right. The red question mark denotes a weak additional extragradient in lane 4. (f) PEL-like neoplasia in a C.vlL6iMyc mouse. Presented to the left is a photograph of a tumor-bearing mouse containing $\sim 4 \mathrm{ml}$ of hemorrhagic ascites. The Wright-Giemsa-stained cytofuge specimen to the right (bar, $20 \mu \mathrm{m})$ includes a cluster of tumor cells that consists mainly of malignant plasmablasts and plasma cells. The cells are large, basophilic and usually contain one eccentric nucleus. Bi-nucleated cells occur occasionally. (g) Ki67 immunostain of plasmablastic neoplasm in a C.vlL6iMyc mouse. Proliferation index is $70-75 \%$. Note that this neoplasm and the tumors displayed in $\mathbf{c}$ and $\mathbf{f}$ share unifying features of human KSHVassociated PBL, PBM and PEL. These include a very aggressive clinical course and morphology of diffuse, cohesive proliferations of cells that resemble immunoblasts with varying degrees of plasmacytoid differentiation and frequent mitotic figures. Because the diagnostic distinction of the human disease entities depends in large measure on the broader clinical-pathologic context, for example, site of disease involvement, presence of immunodeficiency and infection with $\mathrm{KSHV}^{15}$ it is difficult if not impossible to identify accurate counterparts of these entities in the mouse model. H\&E, hematoxylin and eosin.

human IL-6 plays an important role in flares of KSHV-associated $M C D^{10}$ may be corroborated by the assessment of VIL- 6 TG mice that are either proficient for mouse IL- $6\left(\mathrm{mlL}-6^{+}\right)$or deficient of it $\left(\mathrm{mlL}-6^{-}\right)$. The latter mice can be readily generated by transferring the vIL6 transgene to $\mathrm{C}$ mice that are homozygous for a null allele of mIL-6., 13 Furthermore, adoptive transfer of vIL6iMyc-TG $B$ cells to $\mathrm{mlL}-\sigma^{+}$or $\mathrm{mlL}-\sigma^{-}$hosts may permit one to determine whether mlL-6 production in the tumor microenvironment is as important for vIL-6-driven plasmablastic tumors as it is for Myc-driven plasma cell tumors. ${ }^{13}$ To facilitate this research and enhance our understanding of vlL-6-promoted B-cell disorders, strain C.vIL6 has been made available to the scientific community via the Jackson Laboratory (Bar Harbor, ME, USA) sperm cryopreservation and strain recovery program.

\section{CONFLICT OF INTEREST}

The authors declare no conflict of interest.

\section{ACKNOWLEDGEMENTS}

The technical assistance of Shelby Foor and Casey De Hoedt is gratefully acknowledged. This work was supported in part by NIH Pre-doctoral Training Grant T32 Al007485 to TRR; NIH Hematology Training Grant T32 HL007344 to VST; R01CA151354 from the NCI to SJ; and $\mathrm{NCl}$ Core Grant P30CA086862 in support of the Holden Comprehensive Cancer Center. We dedicate this Letter to the late Dr Kristen Ness Schwickerath, a young, talented immunologist and mother of a 6-year-old who contributed to this cancer project but lost her own fight against cancer before the project could be completed.

TR Rosean $^{1,4}$, CJ Holman ${ }^{1,4}$, VS Tompkins ${ }^{1}$, X Jing $^{1}$, MD Krasowski ${ }^{1}$ $\mathrm{S}$ Rose-John ${ }^{2}$ and S Janz ${ }^{1,3}$

${ }^{1}$ Department of Pathology, University of lowa (UI) Carver College of Medicine, lowa City, IA, USA;

${ }^{2}$ Institute of Biochemistry, Christian-Albrechts-University, Kiel, Germany and

${ }^{3}$ UI Holden Comprehensive Cancer Center, lowa City, IA, USA E-mail: siegfried-janz@uiowa.edu ${ }^{4}$ These authors contributed equally to this work.

\section{REFERENCES}

1 Carbone A, Gloghini A, Serraino D, Spina M. HIV-associated Hodgkin lymphoma. Curr Opin HIV AIDS 2009; 4: 3-10.

2 Du MQ, Bacon CM, Isaacson PG. Kaposi sarcoma-associated herpesvirus/human herpesvirus 8 and lymphoproliferative disorders. J Clin Pathol 2007; 60: 1350-1357.

3 Soulier J, Grollet L, Oksenhendler E, Cacoub P, Cazals-Hatem D, Babinet P et al. Kaposi's sarcoma-associated herpesvirus-like DNA sequences in multicentric Castleman's disease. Blood 1995; 86: 1276-1280.

4 Dupin N, Diss TL, Kellam P, Tulliez M, Du MQ, Sicard D et al. HHV-8 is associated with a plasmablastic variant of Castleman disease that is linked to HHV-8-positive plasmablastic lymphoma. Blood 2000; 95: 1406-1412.

5 Cesarman E, Chang Y, Moore PS, Said JW, Knowles DM. Kaposi's sarcomaassociated herpesvirus-like DNA sequences in AIDS-related body-cavity-based lymphomas. N Engl J Med 1995; 332: 1186-1191.

6 Suthaus J, Stuhlmann-Laeisz C, Tompkins VS, Rosean TR, Klapper W, Tosato G et al. HHV-8-encoded viral IL-6 collaborates with mouse IL-6 in the development of multicentric Castleman disease in mice. Blood 2012; 119: 5173-5181.

7 Anderson PN, Potter M. Induction of plasma cell tumours in BALB-c mice with 2,6,10,14-tetramethylpentadecane (pristane). Nature 1969; 222: 994-995.

8 Zhang K, Kagan D, DuBois W, Robinson R, Bliskovsky V, Vass WC et al. Mndal, a new interferon-inducible family member, is highly polymorphic, suppresses cell growth, and may modify plasmacytoma susceptibility. Blood 2009; 114: 2952-2960.

9 Kovalchuk AL, Kim JS, Park SS, Coleman AE, Ward JM, Morse HC 3rd et al. IL-6 transgenic mouse model for extraosseous plasmacytoma. Proc Natl Acad Sci USA 2002; 99: 1509-1514.

10 Polizzotto MN, Uldrick TS, Wang V, Aleman K, Wyvill KM, Marshall V et al. Human and viral interleukin-6 and other cytokines in Kaposi sarcoma herpesvirusassociated multicentric Castleman disease. Blood 2013; 122: 4189-4198.

11 Tolani B, Gopalakrishnan R, Punj V, Matta H, Chaudhary PM. Targeting Myc in KSHV-associated primary effusion lymphoma with BET bromodomain inhibitors. Oncogene 2014; 33: 2928-2937.

12 Lee HR, Doganay S, Chung B, Toth Z, Brulois K, Lee S et al. Kaposi's sarcomaassociated herpesvirus viral interferon regulatory factor 4 (vIRF4) targets expression of cellular IRF4 and the Myc gene to facilitate lytic replication. J Virol 2014; 88: 2183-2194.

13 Rosean TR, Tompkins VS, Olivier AK, Sompallae R, Norian LA, Morse HC 3rd et al. The tumor microenvironment is the main source of IL-6 for plasma cell tumor development in mice. Leukemia 2015; 29: 233-237. 
14 Duncan K, Rosean TR, Tompkins VS, Olivier A, Sompallae R, Zhan F et al. (18)F-FDG-PET/CT imaging in an IL-6- and MYC-driven mouse model of human multiple myeloma affords objective evaluation of plasma cell tumor progression and therapeutic response to the proteasome inhibitor ixazomib. Blood Cancer $J$ 2013; 3: e165.

15 Thakral C, Thomas L, Gajra A, Hutchison RE, Ravizzini GC, Vajpayee N. Plasmablastic lymphoma in an immunocompetent patient. J Clin Oncol 2009; 27: e78-e81.
This work is licensed under a Creative Commons Attribution 4.0 International License. The images or other third party material in this article are included in the article's Creative Commons license, unless indicated otherwise in the credit line; if the material is not included under the Creative Commons license, users will need to obtain permission from the license holder to reproduce the material. To view a copy of this license, visit http://creativecommons.org/licenses/ by/4.0/

Supplementary Information accompanies this paper on Blood Cancer Journal website (http://www.nature.com/bcj) 